\title{
LE PRESIDENT MOBUTU ET \\ LE PREMIER MINISTRE SOUS L'ACTE CONSTITUTIONNEL DE LA TRANSITION: DUEL OU DUO?
}

\section{Jean-Louis ESAMBO KANGASHE}

Université de Kinshasa

Faculté de Droit

B.P. 204, Kinshasa XI

République Démocratique du Congo

DOMAINE DE RECHERCHE: droit constitutionnel et administratif

\section{SUMMARY}

\section{PRESIDENT MOBUTU AND THE PRIME MINISTER UNDER THE} TRANSITIONAL CONSTITUTIONAL ACT: DUEL OR DUO?

Since the beginning of the democratic process in Mobutu's Zaire, in April 1990, a judicial and political debate has continued to divide the opinion on the power sharing within the executive.

The Transitional Constitutional Act, a result of the political negotiations of the Palais du Peuple, was aimed at resolving the competence conflicts between the 
President of the Republic and the Prime Minister. This constitutional framework, however, was unable to avoid neither the possibility of a "duel" nor the avatars of an alleged "duo" between the two main representatives of the executive function.

In the meantime, the frequent violations of the constitution by the head of state have shown that power sharing within a parliamentary system could not come to a compromise with the power practice under Mobutu.

KEY WORDS: President, politics, Prime Minister, constitution, Zaire

\section{INTRODUCTION}

Le 4 avril 1994, le Président Mobutu avait promulgué l'Acte Constitutionnel de la Transition. Aucun n'a vu dans cette décision, une certaine volonté de l'ancien Chef de l'Etat de s'impliquer "résolument" dans la recherche d'une solution à l'épineux problème du dédoublement des textes constitutionnels de la Transition.

La lecture attentive de ce texte fait clairement apparaître que le constituant de 1994 avait instauré pendant la période de la Transition un régime parlementaire marqué par le bicéphalisme de l'Exécutif et la collaboration des pouvoirs. Cette forme de Gouvernement est caractérisée notamment par l'irresponsabilité du Chef de l'Etat (art. $75 \mathrm{al}_{1}$ et 92). Dans un tel système, c'est le Gouvernement qui assure la pleine gestion des affaires de l'Etat. Faut-il, à cet égard, se féliciter que par rapport au régime de la deuxième République, ce texte ait réussi à écarter le Président Mobutu de la gestion de la Res publica.

Parallèlement au satisfecit apparent que l'on pouvait avoir de la présentation théorique du régime politique prévu par l'Acte Constitutionnel de la Transition, il importe de se poser une question: le Président Mobutu dont la longue histoire politique fut mêlée à celle du Congo indépendant était-il en raison de son tempérament disposé à jouer le rôle d'un Chef de l'Etat symbolique chargé d'inaugurer les chrysanthèmes?

En d'autres termes et en se fondant sur l'adage le trône n'est pas un fauteuil vide, le Maréchal Mobutu ne risquait-il pas, en tant que "garant de la Nation", de s'investir personnellement dans la gestion des affaires publiques, affirmant ainsi son autorité directe sur le Gouvernement? 
En tout état de cause, le respect de l'Acte Constitutionnel de la Transition (art. 37 al. 3) par le Chef de l'Etat et le Chef du Gouvernement, notamment en ce qui concerne les attributions dévolues à chacun d'eux, était de nature à apaiser les esprits et entraîner un fonctionnement harmonieux de l'Exécutif pendant la Transition.

Dans cette hypothèse, on ne pouvait s'empêcher d'affirmer qu'entre le Président de la République et le Premier Ministre, il s'était formé un duo. Le contraire ferait penser à un duel auquel les deux personnalités ont dû se livrer. Dans ce cas, une question demeure posée: même s'il pouvait y avoir dualité entre le Chef de l'Etat et le Chef du Gouvernement dans la gestion des affaires publiques, il y avait lieu de se demander si cette situation était susceptible d'entraîner la rupture entre les deux hommes.

Concrètement, il s'agit de mesurer la portée et le degré de cette dualité dans le processus de transition vers un Etat démocratique.

Dans cette perspective, il importe d'axer nos réflexions sur deux points essentiels: l'examen d'une série d'hypothèses possibles à un duel entre le Chef de l'Etat et le Chef de l'Exécutif et la possibilité d'un duo au sein de l'Exécutif. Cette démarche nous a permis de mesurer la nature et la portée du principe de collaboration des pouvoirs qui, il y a peu, fut érigé en règle d'or pendant la Transition.

\section{LES VIRTUALITES D'UN DUEL AU SEIN DE L'EXECUTIF}

Tirant les leçons des méandres, des turbulences et des incertitudes qui caractérisèrent le processus de transition notamment dans les rapports entre le Chef de l'Etat et le Chef du Gouvernement au sujet de la gestion des affaires publiques, la classe politique s'était, lors des négociations politiques du Palais du Peuple accordé sur un principe la collaboration des pouvoirs. La nécessité d'un tel mécanisme fut d'autant plus ressentie qu'elle était à même d'éviter tout conflit de compétence au sommet de l'Etat.

Mais au-delà de cette préoccupation au demeurant légitime, il restait qu'une autre explication méritait d'être donnée. Il ne faisait, en effet, aucun doute que 1 'affaiblissement et la désorganisation de l'opposition dite radicale ainsi que les rapports des forces après les travaux de la Conférence Nationale Souveraine, furent autant de facteurs qui ont notamment fait basculer les données du pouvoir en faveur de la mouvance présidentielle. Au sein de l'Exécutif, en effet, la collaboration fut particulièrement exigée entre le Président de la République et 
le Premier Ministre dans les domaines de la Défense Nationale et des affaires étrangères (art. 75 al. 3). L'attribution de ces deux postes ministériels devait également obéir au principe du "partage équitable et équilibré" du pouvoir entre les "deux familles politiques" $\mathrm{d}$ 'alors.

Toujours en rapport avec la collaboration du pouvoir, il a été entendu qu'une famille politique ne pouvait occuper ni gérer seule un secteur de la vie nationale.

Ce vœu fut également formulé par la Conférence Nationale Souveraine qui recommanda au Chef de l'Etat et au Chef du Gouvernement de maintenir de manière permanente des consultations informelles sur toutes les questions d'intérêt général. Il s'agissait pour les deux personnalités de recourir souvent à la pratique dite du téléphone rouge. Ainsi, en dehors des consultations informelles, la collaboration devrait impérativement se faire par l'entremise du Haut-Conseil de la République-Parlement de Transition.

Mais il ressortait de la situation politique du pays avant l'avènement de l'AFDL au pouvoir certains faits qui ont fait apparaître des signes de fragilité de la collaboration entre le Chef de l'Etat et le Chef du Gouvernement particulièrement en ce qui concerne la Défense Nationale, les Affaires Etrangères et la gestion quotidienne de l'Etat.

\section{Dans le domaine de la Defense Nationale}

Depuis les négociations politiques du Palais du Peuple, la classe politique s'était accordée pour que, la Défense Nationale soit gérée de manière concertée entre le Président de la République et le Premier Ministre.

Aussi, pour préserver la paix sociale, la tranquillité publique et rassurer tout le monde avant les échéances référendaires et électorales projetées, une concertation permanente devait être maintenue dans les rapports entre le Premier Ministre censé être de l'opposition et le Président de la République qui appartenait aux Forces Politiques du Conclave. C'est dans ce cadre que le Chef du Gouvernement fut, à l'instar du Président du Haut-Conseil de la RépubliqueParlement de Transition, membre du Conseil Supérieur de la défense (art. 41 al. 1).

Ainsi, "sur proposition du Gouvernement délibérée en Conseil des Ministres et après avis conforme du Haut-Conseil de la République-Parlement de Transition, le Président de la République nommait, relevait de leurs fonctions et, le cas échéant, révoquait les officiers supérieurs et généraux des Forces Armées et des Forces de l'Ordre, le Conseil supérieur de la défense entendu" (art. 47 al. 2). 
Mais, les nominations et les promotions des Officiers Supérieurs et Généraux des anciennes Forces Armées, des Forces de l'Ordre et de la Garde Civile, intervenues à partir de juin 1994, n'avaient pas respecté la procédure constitutionnelle contraignante en la matière: ces décisions, que l'on a attribué à la seule volonté du Président de la République, ne furent pas l'objet d'une concertation préalable entre le Gouvernement, le Haut-Conseil de la République Parlement de Transition et le Chef de l'Etat.

Jusqu'il y a peu encore, nous avons assisté, depuis le 8 décembre 1996, à la signature par Mobutu d'une série d'ordonnances' ${ }^{1}$ portant restructuration des anciennes Forces Armées zaïroises, spécialement en ce qui concerne le Chef de l'Etat-Major Général, les Chefs d'Etats-Majors et les Commandants des unités spécialisées, ainsi qu'au niveau de la Direction de la Justice Militaire.

Ces ordonnances, qui avaient violé les dispositions impératives de l'article 47, al. 4 de l'Acte Constitutionnel de la Transition, n'ont pas été dénoncées par le Gouvernement et le Parlement de Transition.

Toujours en rapport avec la violation de la Constitution dans la gestion de la Défense Nationale, il y a lieu de signaler que, nonobstant les instructions du Premier Ministre, l'Etat-Major Général des Forces Armées zaïroises d'alors s'était illustré, tout au long de l'année 1995, au recrutement des nouveaux éléments, pendant que l'effectif exact des hommes de troupes n'était pas connu. Si l'on peut affirmer que l'ancien Chef du Gouvernement ne maitrisait pas tous les aspects de la Défense Nationale, on peut se poser la question de savoir si tout cet amalgame d'irrégularités échappait au contrôle du Maréchal-Président.

Aussi, soucieux d'avoir la mainmise sur la conduite de la Transition, en vue justement des échéances référendaires et électorales, Mobutu s'était-il personnellement investi dans la maîtrise des Forces Armées, la Sécurité, les Finances et la Diplomatie, qui ne pouvaient pas être contrôlées par l'Opposition dans un Gouvernement véritablement responsable de la gestion des affaires de l'Etat.

Telles furent, à notre avis, les raisons qui ont justifié certaines décisions prises en violation flagrante de la Constitution dans le domaine de la Défense Nationale. Seulement, était-il fondé de sacrifier le respect des règles constitutionnelles acceptées par tous, au profit d'une allégeance hypothétique à un homme ou à un groupe de personnes?

\footnotetext{
' Il s'agit notamment des ordonnances: $\mathrm{N}^{\circ} 96 / 06 / 062063064,96 / 06 / 062063065,6 / 06 / 062$ 063066 du 08/12/1996 et 97/004 du 08/01/1997.
} 


\section{La collaboration au niveau de la gestion de la Diplomatie}

A l'instar de la Défense Nationale, la diplomatie a été voulue comme étant un domaine sensible dans lequel une famille politique ne pouvait seule contrôler pendant la période de la Transition. La nécessité d'une telle collaboration fut ressentie pour éviter que le Chef de l'Etat et le Chef du Gouvernement ne se livrent à un braconnage diplomatique ${ }^{2}$, étant entendu qu'au Congo, la quête de la légitimité extérieure semble avoir été érigée en programme de Gouvernement.

Le braconnage diplomatique signifie que, lors de sa participation dans différentes conférences et rencontres internationales, le Congo se présente soit avec deux délégations parallèles, soit avec deux points de vue différents entre le Gouvernement et le Président de la République sur une question, soit avec des espions du Gouvernement ou de la Présidence, "simulés" dans la délégation officielle, en vue de suivre ou de contrôler l'action du Gouvernement ou du Président de la République.

Ainsi pour éviter une telle situation, une collaboration permanente devrait être maintenue entre le Président de la République et le Premier Ministre, dans le domaine de la diplomatie. Il y a lieu de relever cependant que cette collaboration a fait défaut, lorsque les troupes françaises se sont installées sur notre territoire dans le cadre de l'opération Turquoise au Rwanda.

Cette pratique en marge de la Constitution explique-t-elle l'affirmation faite sur les antennes de la télévision nationale, le 2 mars 1995, par le professeur VUNDUAWE, ancien Directeur du Cabinet du Président Mobutu, et selon laquelle les grandes questions se traitent, sur le plan diplomatique, par téléphone?

En ce qui concerne le rapatriement des réfugiés Ruandais vers leur pays, alors que le Gouvernement avait fixé le délai butoir au 3 décembre 1995, le Chef de l'Etat laissait entendre, lors de sa participation au Sommet des Pays des Grands Lacs, tenu au Caire du 26 au 29 novembre 1995 sous les auspices de l'ancien Président américain JIMMY CARTER, que cette date, arrêtée après l'adoption d'une résolution par le H.C.R.-P.T., ne le concernait pas.

Aussi, critiquant la position du Président Mobutu, Mgr. Monsengwo avait-il soutenu que le Chef de l'Etat a pris des engagements sans être accompagné $d u$ Ministre des Affaires Etrangères. Par ailleurs, le Chef de l'Etat a pris position

\footnotetext{
${ }^{2}$ Dans ce sens, voir M. DUVERGER, Le système politique français, Paris, P.U.F., 1985, p. 618.
} 
contre une résolution du H.C.R.-P.T. en faveur du retour des réfugiés et contre la position du Gouvernement'.

Cette critique acerbe du Prélat catholique ne fut pas entendue, parce que finalement, c'est la position de Mobutu qui l'emporta. Par ailleurs, quoique fortement controversée dans l'opinion, le limogeage, le 21 janvier 1997, de l'Ambassadeur RAMAZANI BAYA par l'ancien Chef de l'Etat à partir de la France, sans l'intervention du Gouvernement et du Parlement, démontre, s'il en était encore besoin, que le Président Mobutu qui n'a pas entendu se conformer à la Constitution, était loin de garantir la sécurité juridique des citoyens, particulièrement de son diplomate couvert par l'immunité diplomatique.

Il en résulte qu'aussi bien dans le domaine de la Défense Nationale que dans celui de la diplomatie, c'est le Président Mobutu qui était le véritable maître du jeu. Ainsi, le bicéphalisme de l'Exécutif prévu par l'Acte Constitutionnel de la Transition (art. 38) apparaissait inégalitaire et une certaine hiérarchie interne, voire externe, plaçait le Président de la République à la tête de l'Exécutif. Qu'il s'agisse, en effet, du choix du Chef du Gouvernement, de son remplaçant, de la composition du Gouvernement ou de la désignation des responsables de l'armée, le Chef de l'Etat avait une grande influence et une suprématie sur le Premier Ministre. Cette situation fut également apparue même dans la gestion quotidienne des affaires de l'Etat.

\section{LA CONCERTATION DANS LA GESTION DES AFFAIRES DE L'ETAT}

Tirant les leçons de la gestion du pays sous la deuxième République, la classe politique a, tant au cours des travaux de la Conférence Nationale Souveraine qu'à ceux des négociations politiques du Palais du Peuple, levé une option fondamentale: le Chef de l'Etat ne devait plus gérer les affaires de l'Etat. Cette tâche incombait au Gouvernement qui en répondait devant le Haut-Conseil de la République, Parlement de Transition.

Mais, avec la volonté qu'afficha le Maréchal-Président de s'impliquer personnellement dans la gestion de l'Etat, il y avait lieu de craindre un retour de manivelle car après avoir promis de se conformer à la Constitution, Mobutu a fini, comme à l'accoutumée, par s'en écarter: il a vite abandonné le rôle d'arbitre que lui conférait la Constitution (art. 39) pour devenir un véritable Chef de Gouvernement.

\footnotetext{
${ }^{3}$ Extrait tiré de la Conférence de Presse tenue le 14 mars 1996 et reproduite de Marie-France GROS par le quotidien LE PALMARES, dans son numéro 643 du 18 mars 1996, sous le titre De Bruxelles, Mgr. MONSENGWO accuse MOBUTU , p. 8.
} 
Il s'en est suivi qu'en voulant interpréter la Constitution à sa manière, le Maréchal qui s'était longtemps considéré comme garant de la Nation a dû modifier la répartition constitutionnelle des compétences entre lui et le Premier Ministre KENGO porté aux affaires grâce à la majorité que Mobutu détenait au Parlement de Transition ${ }^{4}$.

Il en découle qu'en pareilles circonstances, le Gouvernement maîtrisait très peu la durée de son mandat d'autant plus que le Président de la République pouvait, à tout moment, mettre en mouvement les mécanismes prévus par les dispositions de l'article 69, al. 3 de l'Acte Constitutionnel de la Transition. Cet article était ainsi libellé: les décisions relatives à la mise en accusation du Président de la République, à la censure du Gouvernement et à la modification de l'Acte Constitutionnel de la Transition, sont prises, en cas de recours au vote, à la majorité des trois quarts des membres qui composent le Haut-Conseil de la République-Parlement de Transition.

Il en a été ainsi, lorsque accusant le Gouvernement KENGO de gérer avec légèreté la guerre dite de l'est du pays, la mouvance présidentielle majoritaire au Parlement de Transition, s'était décidée, avec le concours d'une bonne partie de l'Opposition, de faire tomber le cabinet par une motion de censure le 18 mars 1997.

Quoi qu'entachée d'irrégularités, la décision du Parlement fut sans appel et ce, malgré les tentatives de Monsieur KENGO de faire infléchir la position du Président de la République qui n'était certainement pas étranger à cette décision. En prenant acte du retrait de la confiance du Gouvernement par HCR-PT, le Maréchal Mobutu entérinait automatiquement la démission du cabinet.

L'incertitude sur la durée du mandat du Gouvernement plaçait le Président de la République dans une position confortable. Ainsi, dès qu'une mesure prise par le Gouvernement ne le satisfaisait pas, le Président Mobutu faisait souvent connaître ses réserves, mieux sans désapprobation, alors qu'une concertation préalable entre les deux Institutions aurait permis d'éviter les divergences de vues auxquelles on assistait et qui ont donné l'impression qu'un duel s'était installé au sommet de l'Exécutif.

S'agissant particulièrement de la mesure de privatisation de certaines entreprises publiques décidée par le Gouvernement KENGO, il y a lieu de signaler que, pendant que l'Exécutif la trouvait incontournable, le Président Mobutu la qualifiait d'inopportune parce qu'initiée par un Gouvernement provisoire non issu des élections. En définitive, le processus de privatisation fut mis en

\footnotetext{
${ }^{4}$ Déclaration faite en septembre 1994 sur Affirma $n^{\circ}$ l, lors de l'émission Une heure pour convaincre, après le Sommet de Biarritz en France.
} 
veilleuse; comme pour dire que dans cette guerre, ce fut le Président de la République qui en était sorti vainqueur.

Par ailleurs, alors que l'Acte Constitutionnel de la Transition donnait pouvoir au Gouvernement de proposer à la nomination présidentielle, après avis conforme du Haut-Conseil de la République-Parlement de Transition, des fonctionnaires de l'administration, tels que les Directeurs et les Secrétaires Généraux de la Fonction Publique (art. 47, al. 2), le Président Mobutu prit, en date du 31 mars 1995, une série d'ordonnances portant nominations et promotions des Inspecteurs de l'Enseignement Primaire, Secondaire et Professionnel, en violation des dispositions de l'article 47, al. 1 de la Constitution. C'est ce qui a expliqué la réaction du Premier Ministre dans la lettre adressée, en date du 7 avril 1995, au Président intérimaire de l'ancien Parlement de Transition et dans laquelle il fut sans équivoque: ces ordonnances, disait-il, ne sont pas le fait du Gouvernement, qui n'en a pris connaissance qu'à l'occasion de leur publication.

$\mathrm{Si}$, pour une fois, on pouvait se féliciter de la réaction du Premier Ministre KENGO face à la résurgence de cette pratique anticonstitutionnelle longtemps décriée, il y avait lieu, de se poser une question: en s'adressant au Président du Parlement de Transition, le Premier Ministre qui a cru voir ces ordonnances annulées, ne s'était-il pas trompé de cible

Devant cette tentative de résurgence des pratiques de la deuxième République, d'aucuns se sont demandé si la collaboration des pouvoirs sous Mobutu n'était pas mal partie pendant la période de Transition.

Ce retournement de la situation imputable à la personnalité du MaréchalPrésident et à l'affaiblissement de l'Opposition avait, notamment favorisé une certaine dualité au sommet de l'Exécutif.

Mais il importe de se demander si cette dualité était de nature à compromettre les rapports entre Mobutu et KENGO au point d'entraîner la rupture ou la conflictualité entre les deux hommes.

\footnotetext{
5 N. MUKIAP, Annulation des ordonnances. Qui a induit la Présidence en erreur? In: Palmarès $n^{\circ} 387$ du 21 avril 1995, p. 7.
} 


\section{LES AVATARS D'UN DUEL ENTRE LE CHEF DE L'ETAT ET LE CHEF DU GOUVERNEMENT}

Depuis le déclenchement du processus démocratique au Congo/Kinshasa, la vie politique fut marquée par la canalisation des aspirations populaires autour de deux camps dirigés par le Président Mobutu et Monsieur Etienne TSHISEKEDI ${ }^{6}$. Cette restructuration du paysage politique avait rendu nécessaire la recherche du repositionnement dans les deux camps.

La bipolarisation de la vie politique qui s'en est suivie atteignit son paroxysme avec notamment la publication du Gouvernement TSHISEKEDI, issu de la Conférence Nationale Souveraine, à qui on reprochait d'avoir été monocolore et formé, pour la plupart, de novices dans le monde politique. Peut-être que le Premier Ministre voulait apporter une touche personnelle dans la composition de son Gouvernement, avec la présence d'hommes nouveaux pour donner un nouveau souffle dans la gestion des affaires de l'Etat. Cependant, le Chef du Gouvernement avait oublié une autre dimension importante du problème: le poids politique fort minime et l'inexpérience de certains membres du Gouvernement avaient discrédité le cabinet dont l'homogénéité ne fut pas préservée.

Aussi, n'ayant pas trouvé leur compte dans le partage du gâteau, certains membres influents de l'Opposition avaient-ils décidé de se désolidariser de leur Leader pour entretenir des contacts informels avec la Mouvance Présidentielle. Ainsi, on assista à l'époque de révocations, de défections et de débouchages au sein de l'Opposition, ce qui a permis à Mobutu de reprendre en main la gestion de la Transition.

A cet égard, il n'est pas exagéré d'affirmer que l'Opposition dite Radicale nous a donné l'impression d'avoir été essoufflé et manquait de stratégies efficaces pour refaire son unité. Pas plus qu'elle ne disposait pas de véritable politique d'anticipation devant les grands événements politiques du pays, l'Opposition congolaise s'enfonçait dans des querelles internes parfois stériles liées notamment à la gestion des ambitions.

S'agissant justement de la gestion des ambitions, on note que, nonobstant la révocation illégale du Gouvernement TSHISEKEDI, issu de la C.N.S., c'était au sein du Parti du Premier Ministre que le Chef de l'Etat nomma comme Premier Ministre, Monsieur Faustin BIRINDWA, ancien responsable du Secrétariat

\footnotetext{
${ }^{6}$ G. IWELE, Mgr. MONSENGWO, Auteur et témoin de l'histoire. Louvain-La-Neuve, Bruxelles, DUCULOT, 1995, p. 195.
} 
Technique de l'Opposition. Ce qui conduisit au dédoublement institutionnel pendant la Transition?

Mais à l'issue des travaux de concertations politiques du Palais du Peuple, un Protocole d'Accord et un Arrangement Particulier relatifs au partage équitable et équilibré du pouvoir pendant la Transition mirent d'accord la classe politique sur certains points. En ce qui concerne les principes généraux d'orientation de la Transition, les deux délégations avaient convenu de la nécessité d'instaurer un climat de réconciliation nationale. Cette réconciliation couvrait les axes suivants:

1. classe politique, en général;

2. Mobutu-Tshisekedi, en particulier;

3. régions du Zaïre;

4. Kasaïens et Katangais (art. 2 de l'Arrangement Particulier).

L'article 3 du même texte précisait que: les deux délégations conviennent, en conséquence, que la crise Gouvernementale ne doit se résoudre:

1. contre les Forces Politiques du Conclave, ni contre l'Union Sacrée de l'Opposition Radicale et Alliée;

2. contre Monsieur MOBUTU SESE SEKO, ni contre Monsieur Etienne TSHISEKEDI;

3. au détriment de l'intérêt supérieur de la Nation;

4. au détriment de la paix civile ou en compromettant celle-ci au Katanga et dans les autres Régions du Zaïre.

Il en résulte que la classe politique avait, officiellement, reconnu qu'aucune solution à la crise Gouvernementale ne pouvait durablement être envisagée au profit de Mobutu et contre TSHISEKEDI. Mais, en décidant d'élire, au mépris total des dispositions de l'article 78 de l'Acte Constitutionnel de la Transition, un Premier Ministre, le Haut-Conseil de la République-Parlement de Transition avait résolu de favoriser la persistance de la crise Gouvernementale.

\footnotetext{
${ }^{7}$ Le Secrétariat Technique de l'Opposition était chargé notamment de concevoir et de préparer les stratégies politiques de l'Opposition.
} 
Dans cette perspective, il y a lieu de se poser la question sur les facteurs qui ont amené le Parlement de Transition à interpréter, en violation de l'article 102, al. $1^{8}$, la Constitution. En d'autres termes, l'arrivée de Monsieur Léon KENGO WA DONDO à la tête du Gouvernement de Transition avait-elle obéi aux préoccupations d'ordre juridique ou politique?

Saisie par la requête du 26 janvier 1995, introduite par l'USOR et Alliés, Etienne TSHISEKEDI WA MULUMBA et consorts, en vue d'obtenir l'annulation, pour excès de détournement de pouvoir et, partant, pour illégalité, des ordonnances présidentielles $n^{\circ} 94 / 039$ du 16 juin 1994 et $94 / 042$ du 6 juillet 1994, portant respectivement investiture d'un Premier Ministre, en la personne de Monsieur KENGO WA DONDO, et nomination des membres du Gouvernement, la Haute Cour s'était, dans son arrêt R.A. 320 du 21 août 1996, contentée de renvoyer dos à dos les parties, prétextant qu'elle ne pouvait connaître d'une requête contre les actes de Gouvernement.

Cette décision qui nous semble avoir été dictée par des considérations politiques, ne fera pas l'objet d'une analyse approfondie dans le cadre restreint de cette étude. Il n'empêche que, nonobstant cette position fort décevante de la Cour Suprême de Justice, l'ancien Parlement de Transition qui n'avait pas le pouvoir d'interpréter la Constitution, ne pouvait pas faire la lecture littérale de l'article 78; ce qui l'a amené à se saisir du dossier et de statuer ultra petita.

En effet, alors que l'alinéa 2 de cet article prévoyait la possibilité, pour le HautConseil de la République-Parlement de Transition, de se saisir du dossier audelà de dix jours à compter de la promulgation de l'Acte sous examen, ce texte n'avait pas autorisé au Parlement de Transition de se substituer à la Cour Suprême de Justice; de même qu'il ne lui avait pas donné le pouvoir de régler le problème de l'animateur du Gouvernement en dehors du consensus prévu à l'article 69, al. 2.

Pas plus que la possibilité d'élire un Premier Ministre était proscrite lors des négociations politiques, le Haut-Conseil de la République-Parlement de Transition avait violé son propre texte en procédant à l'enregistrement des candidats Premiers Ministres bien avant même le terme du délai prévu par l'article 78. En tout état de cause, l'élection de Monsieur KENGO à la tête du

\footnotetext{
${ }^{8}$ Cet article était ainsi libellé: Sans préjudices des autres compétences qui lui sont reconnues par le présent Acte ou par les lois, la Cour Suprême de Justice connaît des recours en appréciation de la constitutionnalité des lois et des actes ayant force de loi, ainsi que des recours en interprétation du présent Acte, des pourvois en cassation, formés contre les décisions rendues en dernier ressort par les Cours et Tribunaux et des recours en annulation des actes et décisions des autorités centrales de la République, ainsi que des contestations nées des élections et du référendum.
} 
Gouvernement avait obéi plus aux préoccupations politiques qu'elle n'a été conforme à la Constitution.

Au plan politique, il faut signaler qu'à la suite de la révocation, le 21 octobre 1991, du Gouvernement TSHISEKEDI issu des Accords du Palais du Marbre I, le Chef de l'Etat avait laissé entendre que, jamais, il ne fera un second SOGLO en Afrique'.

Ainsi, du fait que Monsieur TSHISEKEDI était présenté par une certaine opinion pour potentiel challenger du Maréchal-Président aux élections présidentielles, il était presque évident que Mobutu s'en débarrasse.

Dans cette perspective, il convient de rappeler que l'histoire politique du Congo nous enseigne que, sous la première République, le refus de l'alternance a amené le Chef de l'Etat KASA-VUBU à limoger Premiers Ministres LUMUMBA et TSHOMBE.

$\mathrm{Si}$, vis-à-vis de LUMUMBA, KASA-VUBU craignait un éventuel coup d'état communiste dont il risquait d'être victime ${ }^{10}$, à l'égard de TSHOMBE, en revanche, le Chef de l'Etat voulait se débarrasser d'un adversaire de taille vers la course à la magistrature Suprême. L'ambition déclarée de Moïse TSHOMBE d'accéder, à tout prix, à la Présidence de la République avec l'appui de la Belgique et des Etats-Unis, ainsi que sa popularité, tant sur le plan interne qu'externe, furent autant de facteurs qui précipitèrent la chute de son Gouvernement ${ }^{1}$. Indépendamment de la complexité de sa déchéance à la tête du HCR-PT, il semble que c'est en application de cette stratégie que Mgr. Monsengwo a été évincé de la présidence du Parlement de Transition.

Mais, affaibli par la maladie et confronté à l'échec de la contre-offensive de son armée face à la progression de l'armée de l'A.F.D.L. conduite par LaurentDésiré KABILA, le Maréchal-Président, qui a été forcé de quitter le pouvoir le 16 mai 1997, a, auparavant, tendu la main à Mgr. Monsengwo, invitant ce dernier à accepter de revenir à la tête du HCR-PT en vue de négocier avec la rébellion pendant qu'en 1995 , le prélat catholique fut déchu de la présidence du Parlement de Transition sous son instigation. Mgr. Monsengwo qui avait

\footnotetext{
9 Allusion faite ici à Cicéphore SOGLO qui, nommé Premier Ministre en 1991 par le Président Matthieu KEREKOU, s'était porté candidat à la Présidence de la République et avait remporté les élections au dépens du Président KEREKOU.

${ }^{10}$ E.O. DJELO, Impact de la coutume sur l'exercice du pouvoir en Afrique. Le cas du Zä̈re, Ottignies, Louvain-La-Neuve, Le Bel Elan Editeur, 1990, p. 32.

1 Analyses et documents relatifs au processus qui a conduit à la fin du Gouvernement TSHOMBE, in: "Dossiers CRISP", n 47 du 20 octobre 1965, p. 5 et 9.
} 
vraisemblablement accepté d'assumer les fonctions de Président intérimaire en cas de démission ou d'empêchement du Chef de l'Etat (art. 53 et 54, al. 3) s'était ravisé le 11 mai 1997 lorsque, au cours d'une conférence de presse à Bruxelles, il posait deux conditions pour jouer ce rôle: une garantie nationale et internationale et l'acceptation par toute la classe politique de l'ordre institutionnel de la C.N.S. et de son projet de société.

La position ambiguë de l'Archevêque de Kisangani, que d'aucuns avaient qualifié de traite pour avoir accepté de venir au secours d'un régime décédant, fut confrontée à une hostilité de l'Opposition armée et non armée. Finalement, le scénario n'a pas payé, car, le 17 mai 1997, la Capitale congolaise fut conquise par l'A.F.D.L. et son Président s'était autoproclamé le Chef de l'Etat de la République Démocratique du Congo.

Il en résulte que la période de Transition sous Mobutu ressemblait à la première, voire même à la deuxième République, dans la lutte acharnée que les Chefs d'Etat n'avaient cessé de mener contre ceux que VANDERLINDEN a appelé les

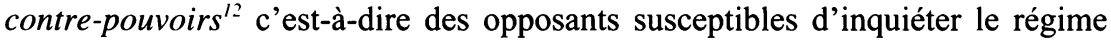
en place.

Quoi qu'il en soit, en accédant à la désignation de Monsieur Léon KENGO WA DONDO à la tête du Gouvernement et ce, au mépris total de l'article 78 susindiqué, le Président de la République était certain d'une chose: l'ancien Chef du Gouvernement ne le gênait pas. Pas plus que Monsieur KENGO refusait d'être concurrent du Maréchal Mobutu aux élections présidentielles, il semble que la tradition Ngbandi enseigne que, par une alliance fraternelle, le Premier Ministre KENGO ne pouvait souhaiter du mal au Président de la République ni se liguer contre lui. Cette situation fut d'autant plus évidente que, lors de son séjour américain en mars 1995, le Premier Ministre avait officiellement déclaré, devant la presse étrangère, qu'il ne sera candidat à aucun poste politique électif à l'issue de la Transition ${ }^{13}$.

N'ayant aucune ambition politique déclarée, le Premier Ministre KENGO fut présenté par une certaine opinion comme la personne la mieux indiquée pour garantir à Mobutu sa réélection à la tête du pays de sorte qu'entre les deux, il s'est formé un duo plutôt qu'un duel.

\footnotetext{
12 J. VANDERLINDEN, La deuxième République, in : "Du Congo au Zaïre" (1960-1980), Essai de Bilan, Bruxelles, Dossiers CRISP, 1980, p. 154.

${ }^{13}$ Cette déclaration qui a été faite le 23 mars 1995, n'a été diffusée sur les antennes de la télévision nationale que deux jours après.
} 
Ironie du sort, le Maréchal-Président ne sera plus candidat à sa propre succession et le M.P.R., dont le fondateur est mort en exil doit revoir ses stratégies.

Aussi, nonobstant des rivalités apparentes, de malentendus passagers que l'on a pu déceler dans les rapports entre Mobutu et KENGO notamment dans la gestion des domaines dits de collaboration, on était loin de considérer que ces avatars ont été de nature à créer la rupture entre les deux hommes au point d'ouvrir la porte vers une collaboration risquée avec l'aide dure de l'Opposition.

L'hypothèse d'un duel au sein de l'Exécutif étant écartée, il restait qu'en décidant de former un duo, Mobutu et KENGO avaient pris l'engagement de ne pas violer le pacte qui les liait depuis des années et ce, au mépris de la réconciliation nationale prévue par le Protocole d'Accord des concertations politiques du Palais du Peuple et l'Arrangement Particulier.

Dans cette perspective, il est permis de s'interroger sur l'héritage dont la population a bénéficié de cette alliance au moment où elle ne cache plus sa détermination à la revendication d'un Etat de droit démocratique.

\section{CONCLUSION}

Depuis l'annonce, le 24 avril 1990, de l'ouverture démocratique, le Congo traverse une crise politique aiguë, essentiellement marquée par l'absence du respect par les hommes politiques des engagements librement pris, de sorte que la période de la Transition qui passe pour la plus longue de l'Afrique tend à obéir à une logique: le refus de l'alternance. A cet égard, il importe de rappeler que la libéralisation de la vie politique amorcée depuis sept ans, a donné lieu à un débat juridique et politique fort controversé au sujet de la répartition des compétences entre le Président de la République et le Premier Ministre.

Mais, les divergences de vues qui avaient opposé la classe politique à propos de l'organisation et la conduite de la Transition ont démontré que la crise qui touche la société congolaise est aussi une crise de démocratie. La longueur de la période de Transition ainsi que la succession des différents Gouvernements à la tête de l'Etat attestèrent que la crise politique congolaise est loin d'être résolue au sens du partage équitable et équilibré du pouvoir et la démocratie tant attendue par la population semble encore au parking. Ainsi, au lieu de connaître des avancées telles que souhaitées par une bonne partie de la population, la période de la Transition tend à suivre un mouvement caractérisé par des tergiversations, des reculades ou mieux, un mouvement marqué par la politique 
d'essais et d'erreurs, déjà connu sous la deuxième République et dont la seule finalité est la conservation du pouvoir.

Toutefois, même si l'exercice de la politique peut parfois obéir à certaines exigences de la conservation du pouvoir on ne peut s'empêcher d'aller frapper à la porte d'ARISTOTE pour lui demander les recettes qu'il avait réservées à la politique comme l'art de gouverner la cité. Mais, gouverner la cité ne suppose-til pas qu'on fasse prédominer l'intérêt général par rapport aux intérêts privés ou partisans? Aussi, le peuple au nom duquel les hommes au pouvoir se targuent de parler, devait-il mériter d'eux un minimum de respect, de protection et de considération.

C'est dans ce climat d'incertitude et de suspicion qu'une partie de la classe politique qui souhaitait le maintien du duo Mobutu-KENGO à la tête de l'Exécutif s'était déjà investie dans la campagne sur l'organisation rapide d'élections au pays. Il importe de souligner que, même si les élections demeurent un des facteurs déterminants à la résolution de la crise politique congolaise, elles ne doivent pas être considérées comme une panacée. L'absence de la réconciliation nationale risque d'être à la base d'une autre crise consécutive aux élections. Dans ce cas, avant les élections pourraient ressembler à après les élections et cela pourrait ouvrir la porte à des contestations des résultats.

S'agissant, en effet, de la violation répétée - mais non sanctionnée - de la Constitution dans le cadre des rapports entre le Président de la République et le Premier Ministre, il convient de signaler qu'elle est fonction de la versatilité des hommes politiques, mais aussi de l'impunité presque permanente; de sorte que le pouvoir judiciaire, qui devait jouer son rôle de pouvoir sanctionnateur, apparaît de plus en plus marginalisé dans la conduite du processus démocratique en cours. Ce qui a fait dire à une certaine opinion que l'indépendance de la justice est plus une lueur qu'une réalité.

Dans cet ordre d'idées, notons que le pouvoir judiciaire nous donne l'impression d'avoir raté le rendez-vous que lui a offert l'histoire. Plus d'une fois, en effet, ce pouvoir a été sollicité pendant la Transition; ce qui lui aurait permis d'affirmer son indépendance vis-à-vis des pouvoirs Législatif et Exécutif, mais ses réactions face à ces sollicitations ont été timides et ont donné la preuve de son impuissance.

Ainsi, même si l'Acte Constitutionnel de la Transition a opté pour un régime parlementaire, une certaine opinion souhaite encore revenir au régime présidentiel ou, mieux, semi-présidentiel, dans lequel le Gouvernement ne sera pas totalement indépendant du Chef de l'Etat. 
$\mathrm{Du}$ fait que la crise politique congolaise est aussi due au manque de culture démocratique et au refus de l'alternance, il importe d'éduquer et de moraliser les hommes politiques dans le sens du respect de leurs propres textes. Parmi les moyens pour atteindre cet objet figurent les efforts que déploient certaines Associations et Organisations Non Gouvernementales, qui s'investissent dans la dénonciation de la Constitution et des principes démocratiques consensuellement arrêtés. C'est dans ce cadre que nous saluons la création, à Kinshasa, d'une association congolaise de Droit Constitutionnel, dont les objectifs peuvent notamment être regroupés autour de:

- la vulgarisation de l'enseignement du Droit Constitutionnel et des principes fondamentaux de la démocratie;

- la connaissance par chaque citoyen, de la Constitution et des lois de la République, en vue de jouer pleinement son rôle de citoyen particulièrement informé de ses droits et de ses devoirs;

- la dénonciation auprès de l'opinion publique, des Institutions Nationales et Internationales, de toute entrave à la démocratie.

On peut, en effet, douter de l'efficacité de telles actions devant une classe politique versatile, sans morale et obnubilée par le gain facile; mais la portée des initiatives ne se mesure et ne s'apprécie qu'au fur et à mesure de leur assimilation par la population concernée. Quoi qu'il en soit, le meilleur moyen d'éduquer et de moraliser notre classe politique reste le souci de faire du pouvoir judiciaire un véritable pouvoir sanctionnateur de toute violation de la Constitution et des lois de la République, quel qu'en soit l'auteur et son rang social. 


\section{BIBLIOGRAPHIE}

DJELO, E.O., Impact de la Coutume sur l'exercice du pouvoir en Afrique, le cas du Zaïre. Ottignies, Louvain-La-Neuve, Le Bel Elan Edition, 1990.

DUVERGER, M., Le système politique français. Paris, P.U.F., 1985.

IWELE, G., Mgr. Monsengwo, Auteur et témoin de l'histoire, Louvain-LaNeuve, Duculot, 1995.

MUKIAP, N., Annulation des ordonnances. Qui a induit la Présidence en erreur? In: "Palmarès $n^{\circ} 387 "$ du 21 avril 1995.

VANDERLINDEN, J., La deuxième République. In: "Du Congo au Zaïre de 1960 à 1980". Bruxelles, Essai de Bilan, Dossiers CRISP, 1980.

Analyses et documents relatifs au processus qui a conduit à la fin $d u$ Gouvernement TSHOMBE. In: "Dossiers C.R.I.S.P.”, n 48 du 20 octobre 1965.

Le Palmarès nº 643 du 18 mars 1996.

L'Acte Constitutionnel de la Transition. 